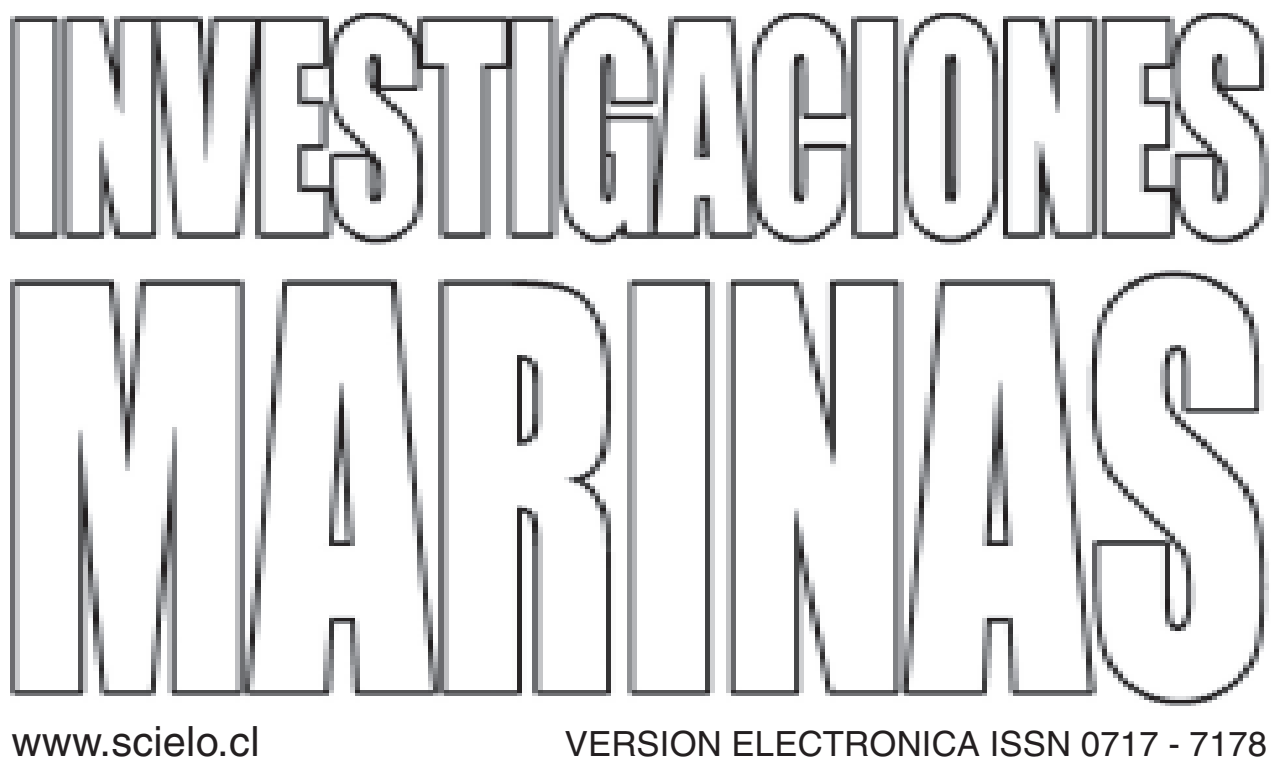

INVESTIGACIONES MARINAS es una revista que considera para su publicación los resultados de investigaciones científicas y tecnológicas originales en ciencias del mar, dando preferencia a los trabajos realizados en el Océano Pacífico Sudoriental y Océano Austral. Los artículos y notas pueden ser publicados en idioma español o inglés.

La revista es de periodicidad semestral y es editada por la Escuela de Ciencias del Mar de la Facultad de Recursos Naturales de la Universidad Católica de Valparaíso.

INVESTIGACIONES MARINAS is a journal for the purpose of publishing results either from scientific or technological research in marine science. The journal will give priority to those manuscripts referring to the Southeastern Pacific or the Southern Ocean. Submission can be made in Spanish or English.

The journal is edited by the School of Marine Sciences, Faculty of Natural Resources of the Catholic University of Valparaiso, and published twice a year. 


\section{EVALUADORES DE LOS TRABAJOS INCLUIDOS EN ESTE NUMERO}

Patricio Aceituno G.

Universidad de Chile

Adriana Gamonal V.

Universidad Católica de Valparaíso

Karin Lohrmann S.

Universidad Católica del Norte

Claudio Palma R.

Universidad de La Serena

Carlos Riquelme S.

Universidad de Antofagasta

Fernando Robles R.

Consultor COI/UNESCO

Sergio Salinas M.

Universidad Católica de Valparaíso
Enrique Dupré M.

Universidad Católica del Norte

Fabián Gómez R.

Universidad Católica de Valparaíso

Armando Mujica R.

Universidad Católica del Norte

Ricardo Pefaur L.

Universidad Católica de Valparaíso

James Robeson C.

Universidad Católica de Valparaíso

José Rutllant C.

Universidad de Chile

Rodolfo Serra B.

Instituto de Fomento Pesquero 


\title{
El Niño-Oscilación del Sur (ENOS) y los frentes fríos que arriban a la región occidental cubana
}

\author{
Benigno Hernández* \\ Centro de Investigaciones Pesqueras, Ciudad de La Habana, Cuba \\ *Dirección actual: CICESE, División de Oceanología, P.O. Box 434844, San Diego \\ CA. 92143-4844 \\ E-mail: bhernan@cicese.mx
}

Recibido: 21 septiembre 2001; versión corregida: 14 enero 2002; aceptado: 2 abril 2002

\begin{abstract}
RESUMEN. Se analiza el periodo invernal del arribo de frentes desde 1923-24 a 1995-96 existiendo una relación positiva directa en los incrementos del arribo de frentes fríos que penetran por la región occidental de Cuba con años donde ocurren eventos ENOS. Las variables meteorológicas más importantes que acompañan a los frentes como la temperatura del aire, presión atmosférica y lluvia, varían con respecto a su media histórica. La temperatura superficial del mar en el golfo de México, es un factor que parece influenciar en un mayor o menor grado el arribo de frentes y la disipación de algunos de ellos cuando atraviesan el mismo. El Análisis de Épocas Superpuestas muestra asociación significativa entre el efecto de los eventos ENOS sobre el aumento en el arribo de frentes fríos a la región occidental de Cuba y algunas variables meteorológicas, por lo que se considera una herramienta estadística apropiada y útil.
\end{abstract}

Palabras claves: frentes fríos, temperatura, presión, lluvia, occidente de Cuba, ENOS.

\section{ENSO and cold fronts arrival in Cuban western region}

\begin{abstract}
Cold front arrivals in winter season is analyzed from 1923-24 to 1995-96 existing a direct positive relationship in the increases of cold fronts in ENSO years in the western region of Cuba. Most important variables in the front's arrivals as air temperature, sea level pressure and rainfall suffer variations with respect to historical average is analyzed. Gulf of Mexico SST is probably factor that seems to influence in a great or smaller fronts arrivals or dissipation of some of then when cross through the Gulf of Mexico. Superposed Epoch Analysis (SEA) offered a significant association degree among ENSO events in the increase of cold fronts arrivals in Cuban western region, for that is considered an appropriate and useful statistical tool.
\end{abstract}

Key words: cold fronts, temperature, pressure, rainfall, western Cuba, ENSO.

\section{INTRODUCCIÓN}

El fenómeno ENOS (El Niño/Oscilación del Sur) (Troup, 1965; Rasmusson y Wallace, 1983), es el resultado de un vasto y complejo sistema de fluctuaciones climáticas entre el océano y la atmósfera y actualmente es considerado como la señal dominante del clima global para escalas de tiempo que oscilan desde meses hasta algunos años (Philander, 1985; Galindo y Centeno, 1989). Este fenómeno se asocia con importantes perturbaciones en los regímenes de lluvias en los trópicos, cuyos resultados son sequías en áreas donde habitualmente llueve y lluvias torrenciales en zonas usualmente desérticas.

El ENOS provoca también cambios en la circulación general extratropical, contribuyendo a que durante el invierno en el hemisferio norte se produzcan situaciones anómalas de tiempo meteorológico, incluyendo severas precipitaciones en el sureste de Estados Unidos, norte de México, golfo de México, estrecho de Florida y occidente de Cuba (Philander, 1983; Philander y Rasmusson, 1985; 
Enfield, 1987, 1989; Ropelewski y Halpert, 1987; Rasmusson et al., 1990; Glantz et al., 1991).

Según White y Downton (1991), hay varios autores que han demostrado la incidencia durante el invierno en el hemisferio norte, de tres patrones que afectan la circulación en el sureste de Estados Unidos, golfo de México y región occidental de Cuba, que son la Oscilación del Sur (IOS) (Ropelewski y Halpert,1987; Bradley et al., 1987); la Oscilación del Atlántico Norte (OAN) (van Loon y Rogers, 1978; Meehl, 1978) y el patrón del Pacífico-Norte América (PNA) (Dickson y Namias, 1976; Horel y Wallace, 1981).

Diversos autores han señalado la influencia del ENOS en variables y eventos hidrometeorológicos que afectan el Caribe noroccidental, tal como Gray (1992) con respecto a la disminución de huracanes en el Atlántico. Meulenert (Instituto de Meteorología, INSMET, Cuba, com. pers.) señaló el aumento de las precipitaciones en distintas regiones de Cuba ante eventos ENOS y Blázquez (1989), reportó el aumento del nivel medio del mar (NMM) en la estación de Siboney al norte de La Habana, en el evento extraordinario ENOS 1982-83. Hernández y Puga (1995) asociaron los efectos del ENOS en la variabilidad hidroclimatológica con capturas de langosta Panulirus argus del golfo de Batabanó, región occidental cubana, mientras que Naranjo (INSMET, com. pers.), realizó estudios sobre el impacto del ENOS en la actividad ciclónica de la cuenca del Caribe. Sin embargo, el estudio de las temporadas invernales asociado a eventos ENOS no ha sido estudiado hasta la fecha.

Durante el efecto de cualquier evento ENOS las presiones medias mensuales de la corriente en chorro subtropical en la región de las Aleutianas-golfo de Alaska, alcanzan valores bajos y los rumbos de las tormentas que penetran en América del Norte provenientes del Pacífico, traen como consecuencia vientos y mareas destructivas hacia la costa de California. La extensión hacia el este y el desplazamiento hacia el sur de la corriente en chorro en Baja California y el golfo de México se asocian con períodos secos y estados borrascosos desde California a Florida y Cuba, en la conocida circulación de Walker (Walker, 1923).

Los frentes fríos o nortes arriban de forma atenuada por la costa noroccidental de Cuba en octubre, para ser casi nulos en mayo y junio. El número de frentes oscila entre 12 y 35 , consideradas como épocas extremas siendo de vital importancia su es- tudio en distintas áreas de la economía de Cuba. Se ha observado una mayor entrada de frentes fríos en temporadas invernales coincidente con años cuando ocurren eventos ENOS. Se procedió a revisar la literatura internacional para conocer más detalles al respecto y no se encontró antecedentes que relacionen los totales por temporadas de frentes fríos con eventos ENOS.

Un adecuado conocimiento de los factores ambientales locales, imprescindibles para el aprovechamiento integral de los recursos naturales no es posible, sin considerar la permanente interacción e impacto de los procesos de escalas mayores que fijan el marco de referencia sobre el que se desarrollan dichas condiciones locales (Rutllant, 1985). Por tanto, dada la importancia que tiene la repercusión del evento ENOS en la salud, bienestar y economía en general, se demuestra estadísticamente la existencia de una mayor probabilidad de entradas de frentes fríos en años ENOS de cualquier clasificación, así como las fluctuaciones en algunas de las variables más importantes que lo acompañan y que influyen en la mitad occidental cubana, considerando para ello el oeste del meridiano $80^{\circ} \mathrm{W}$ en su paso por la región occidental de Cuba.

\section{MATERIALES Y MÉTODOS}

Para el presente trabajo se tuvo en cuenta los registros de frentes fríos (cantidad y clasificación) desde la temporada 1923-24 hasta 1995-96, tomados de Rodríguez et al. (1984), del Departamento de Sinóptica del Instituto de Meteorología, La Habana, Cuba (INSMET) y archivos del autor (Tabla 1).

Las variables que se analizan y acompañan a los frentes son las precipitaciones, temperatura mensual del aire y presión atmosférica a nivel del mar, desde 1923-24 hasta 1995-96, llevados a sus anomalías mensuales y promedios en su período invernal, es decir, entre octubre y marzo. Los datos fueron tomados de la Estación Meteorológica (EM) de Casablanca $\left(23^{\circ} 10^{\prime} \mathrm{N}\right.$ y $\left.82^{\circ} 21^{\prime} \mathrm{W}\right)$, situada a $50 \mathrm{~m}$ de altura en la entrada de la bahía de La Habana. Es una estación de primer orden, posee una serie cronológica de variables meteorológicas y su ubicación a la entrada del golfo de México es capaz de registrar cualquier variación del tiempo en la región occidental de Cuba.

Las precipitaciones en Cuba marcan dos períodos bien definidos, uno lluvioso y otro menos llu- 
Tabla 1. Totales de frentes fríos arribados a Cuba desde 1923-24 hasta 1995-96.

Table 1. Total of cold fronts arrived to Cuba from 192324 up to 1995-96.

\begin{tabular}{|c|c|c|c|}
\hline Año & $\begin{array}{l}\text { Número de } \\
\text { frentes fríos }\end{array}$ & Año & $\begin{array}{l}\text { Número de } \\
\text { frentes fríos }\end{array}$ \\
\hline $1923-24$ & 17 & $1960-61$ & 20 \\
\hline $1924-25$ & 20 & $1961-62$ & 16 \\
\hline $1925-26$ & 27 & $1962-63$ & 21 \\
\hline $1926-27$ & 14 & $1963-64$ & 27 \\
\hline $1927-28$ & 19 & $1964-65$ & 16 \\
\hline $1928-29$ & 19 & $1965-66$ & 23 \\
\hline $1929-30$ & 15 & $1966-67$ & 23 \\
\hline $1930-31$ & 19 & $1967-68$ & 24 \\
\hline $1931-32$ & 15 & $1968-69$ & 28 \\
\hline $1932-33$ & 14 & $1969-70$ & 30 \\
\hline $1933-34$ & 12 & $1970-71$ & 24 \\
\hline $1934-35$ & 16 & $1971-72$ & 23 \\
\hline $1935-36$ & 14 & $1972-73$ & 26 \\
\hline $1936-37$ & 19 & $1973-74$ & 16 \\
\hline $1937-38$ & 19 & $1974-75$ & 21 \\
\hline $1938-39$ & 15 & $1975-76$ & 26 \\
\hline $1939-40$ & 24 & $1976-77$ & 35 \\
\hline $1940-41$ & 20 & $1977-78$ & 28 \\
\hline $1941-42$ & 19 & $1978-79$ & 14 \\
\hline $1942-43$ & 19 & $1979-80$ & 19 \\
\hline $1943-44$ & 16 & $1980-81$ & 20 \\
\hline $1944-45$ & 17 & $1981-82$ & 16 \\
\hline $1945-46$ & 27 & $1982-83$ & 28 \\
\hline $1946-47$ & 22 & $1983-84$ & 23 \\
\hline $1947-48$ & 18 & $1984-85$ & 18 \\
\hline $1948-49$ & 18 & $1985-86$ & 18 \\
\hline $1949-50$ & 19 & $1986-87$ & 18 \\
\hline $1950-51$ & 29 & $1987-88$ & 25 \\
\hline $1951-52$ & 22 & $1988-89$ & 13 \\
\hline $1952-53$ & 15 & $1989-90$ & 18 \\
\hline 195354 & 22 & $1990-91$ & 18 \\
\hline $1954-55$ & 18 & $1991-92$ & 23 \\
\hline $1955-56$ & 26 & $1992-93$ & 25 \\
\hline $1956-57$ & 14 & 1993-94 & 21 \\
\hline $1957-58$ & 26 & 1994-95 & 21 \\
\hline $1958-59$ & 18 & $1995-96$ & 24 \\
\hline $1959-60$ & 24 & & \\
\hline
\end{tabular}

vioso o seco (Lerch, 1982). El primero de mayo a octubre, con un máximo principal en junio y el otro de noviembre a abril. Para este trabajo se consideró el período invernal puesto que los frentes comienzan a llegar en octubre y la lluvia está asociada a la presencia de frentes fríos de corta duración, solamente alterados por numerosos frentes (o tren de frentes fríos) o por la presencia de bajas extratropicales en el golfo de México.
La temperatura del aire en superficie en la región occidental cubana varía en invierno desde los $27^{\circ} \mathrm{C}$ como promedio máximo, hasta los $18^{\circ} \mathrm{C}$ como promedio mínimo, con referencia a la EM de Casablanca. En invierno, las temperaturas mínimas del aire bajan como promedio mensual a $20^{\circ} \mathrm{C}$ en la región occidental y nunca descienden por debajo de $0{ }^{\circ} \mathrm{C}$ en localidades del interior, ya que las aguas del golfo de México son capaces de "calentar" a los más severos, particularmente en el sur. Mientras que el campo bárico en Cuba es dominado en invierno por el sistema migratorio de altas presiones continentales (APC) que imperan en el territorio norteamericano y que suceden a las líneas frontales con buen tiempo, regularmente frío y seco.

Los datos del Índice de Oscilación Sur (IOS) y las diferencias de presión entre la Estación Meteorológica de Darwin y Tahití, fueron ofrecidos por Vernon Kousky de la NOAA, además, de la información de ocurrencias de eventos ENOS. Además, se consideraron otros trabajos, como la cronología de eventos ENOS de Quinn y Neal (1989) y Quinn (1993).

El método estadístico utilizado para relacionar eventos ENOS con los frentes y las variables que le acompañan fue el Análisis de Épocas Superpuestas (Superposed Epoch Analysis, SEA) sugerido por G.A. Maul (FIT, com. pers.) Anexo 1. Es una técnica no-paramétrica que se puede usar para probar significancias estadísticas de asociaciones entre eventos discretos autocorrelacionados, por ejemplo fenómenos hidrometeorológicos extremos, como ENOS, huracanes, frentes, y otros sucesos. Esta prueba fue utilizada anteriormente para el análisis de otros eventos (Panofski y Brier 1965; Haurtwitz y Brier, 1981; Prager y Hoenig, 1989; Hanson et al., 1989; Prager y Hoenig, 1992). En este caso, la prueba es una análoga al t de Student (W) que compara la media obtenida en cada evento clave (0) con los años contiguos, es decir, $(-1,0,+1)$. Una vez obtenido este número se simula por el Método de Monte Carlo, que brinda la probabilidad real que este análisis no es aleatorio, es decir, es más dependiente del evento clave en cuestión. El programa original en lenguaje Fortran (Hoenig et al., 1989), permite computar las pruebas estadísticas mediante simulación aleatoria por el método de Monte Carlo, se compiló en $\mathrm{C}$ y se adaptó para microcomputadora compatible (Alfonso, 1992). 


\section{Breves consideraciones de los frentes fríos que arriban a Cuba}

Se define como un frente frío, la zona o línea de interacción entre una masa de aire frío de origen ártico o polar y una masa de aire caliente de origen tropical. Durante el avance de la masa fría se produce un marcado cambio de tiempo, disminuye la presión atmosférica al paso del frente y varía la dirección y velocidad de los vientos. También se produce una disminución de la temperatura del aire y de la visibilidad asociada a la línea de lluvia durante el paso de la banda frontal. Debido a los fuertes vientos de región norte que se originan en el borde meridional del anticiclón continental -fuente energética que gobierna el avance de la masa de aire frío- se produce un considerable aumento de las marejadas en el mar posterior al paso del frente (Eduardo Rodríguez, Weather Channel, com. pers.), provocando inundaciones, en ocasiones severas en la costa noroccidental de la isla.

En general, la entrada de los frentes fríos se efectúa por la región occidental de Cuba donde llega el $100 \%$ de los frentes y su efecto va disminuyendo a medida que avanzan hacia el interior del país (Fig. 1). $\mathrm{Su}$ arribo comienza a finales de septiembre o principios de octubre hasta abril, aunque en mayo y junio se han reportado frentes débiles o en estado de disipación.

A diferencia de otros países (latitudes medias y altas) en que la clasificación de los frentes fríos tiene como base fundamental el gradiente térmico horizontal, la turbulencia y la cizalladura del viento entre otras variables, en Cuba es el viento y no la temperatura la variable fundamental para la clasificación de los frentes fríos en tipos e intensidad. Según el Instituto de Meteorología de Cuba, la clasificación que se emplea toma como criterio la velocidad del viento y es la siguiente: débiles (frentes cuyos vientos no sobrepasan los $\left.35 \mathrm{~km} \cdot \mathrm{h}^{-1}\right)$, moderados (entre 36 y $55 \mathrm{~km} \cdot \mathrm{h}^{-1}$ ) y fuertes (mayores de 55 $\left.\mathrm{km} \cdot \mathrm{h}^{-1}\right)$.

\section{RESULTADOS}

La serie cronológica de 73 temporadas invernales que han arribado a la región occidental de Cuba muestra que los máximos de arribo de frentes se asocian con años de eventos ENOS (Fig. 2). Solamente en la temporada 1930-31 el total de frentes no sobrepasó la media de 19 frentes, mientras que la otra gran mayoría de escasas entradas se asociaron a años La Niña (Philander, 1985).

Además, se observaron dos períodos extremos en el arribo de frentes, el primero desde 1926-27 hasta 1938-39 (14 años), con totales anuales por debajo del promedio y el segundo, desde 1965-66 hasta 1972-73 (8 años), donde entraron como promedio 25 frentes (Fig. 2). El mínimo de entradas fue en 1933-34 y el máximo en 1976-77 con 12 y 35 frentes respectivamente. Los estadígrafos aplicados a los frentes fríos muestran donde se indica la media, desviación y el recorrido alrededor de los años de influencia ENOS, es decir, una temporada anterior, durante y después $(-1,0,+1)$ de cada evento con un $90 \%$ de confianza (Fig. 3).

La Figura 4 muestra las anomalías de los frentes fríos un año antes, durante y después $(-1,0,+1)$ en cada uno de los eventos ENOS y la clasificación de intensidad señalada por Quinn y Neal (1989) y Quinn (1993), de moderado (-), moderado (+), fuerte y muy fuerte. En la misma se observa una tendencia positiva o de entrada mayor de frentes cuando los eventos son considerados moderados, mientras que se presenta una tendencia negativa en las anomalías de frentes cuando los eventos ENOS son clasificados fuertes.

En este sentido pueden existir, al menos, dos hipótesis esquemáticas de las posibles causas del aumento y disminución del arribo de frentes fríos en las temporadas invernales (octubre-mayo) por la región occidental de Cuba durante años ENOS:

Hipótesis 1. Aumento del arribo de frentes. Al parecer, se intensifica la frontogénesis del continente americano a partir de la presencia más hacia el $\mathrm{S}$ de la corriente en chorro de altas capas de la atmósfera que posibilita ese marcado flujo e intercambio de energías entre el S y el N. Poca influencia o retirada de la región de la cuña del anticiclón del Atlántico del Norte en el invierno, facilitando igualmente, la entrada de los frentes. Disminución en las anomalías de la temperatura superficial del mar en el golfo de México que entran en contacto con la masa de aire fría.

Hipótesis 2. Disminución en el arribo de frentes. Se mantiene la frontogénesis pero no tan intensa del continente americano hacia el sur, ya que la presencia de la corriente en chorro en las altas capas de la atmósfera tiene un marcado flujo con tendencia oeste a este, dificultando la presencia continuada de los arribos de frentes a la región occidental cubana. Aumenta la presencia de la cuña del 


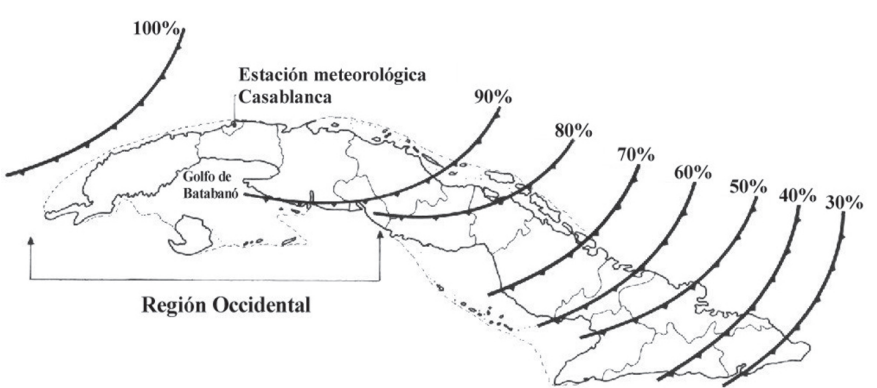

Figura 1. Porcentaje de arribo de los frentes fríos que llegan a las distintas provincias de Cuba (Rodríguez, 1982).

Figure 1. Percentage of cold fronts arriving at the different provinces of Cuba (Rodríguez, 1982).

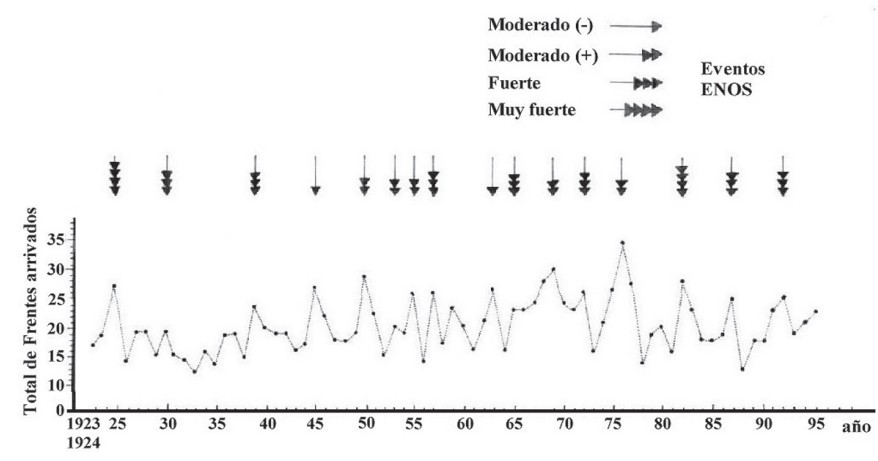

Figura 2. Serie de tiempo y clasificación de los frentes fríos arribados a la región occidental cubana desde 1923-24 hasta 1995-96. las flechas indican los eventos ENOS y su grado de intensidad según Quinn y Neal (1989) y Quinn (1993).

Figure 2. Time series and classification of the cold fronts arriving at the western Cuban region from 1923-24 to 1995-96. The arrows indicate the ENSO events and their intensity according to Quinn and Neal (1989) and Quinn (1993).

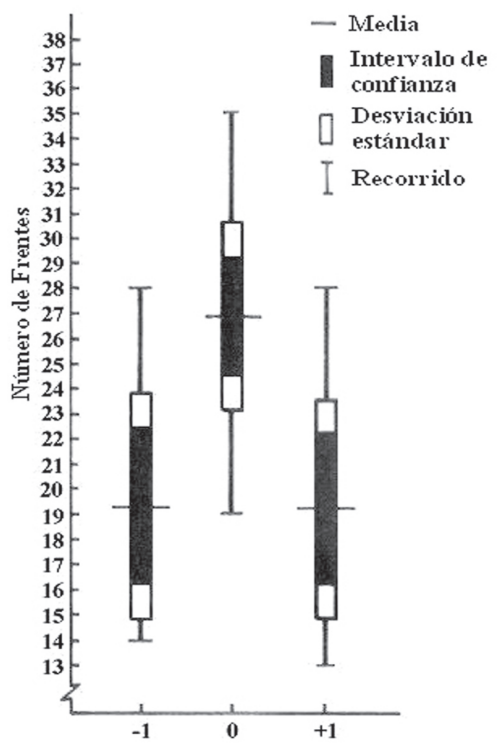

Figura 3. Estadígrafos aplicados a la serie cronológica de frentes fríos desde 1923-24 hasta 199596.

Figure 3. Statistical graphs applied to the chronological series of cold fronts from 1923-24 to 1995-96. anticiclón del Atlántico norte en la región noroccidental del Mar Caribe, incluyendo en ocasiones hasta el golfo de México, provocando un freno a la llegada de los frentes a Cuba, desestabilizándolos en el estrecho de Florida o alrededores. Aumento de las anomalías de temperatura superficial del mar en el golfo de México.

De manera esquemática se muestra la primera hipótesis del aumento en el arribo de frentes donde los flujos turbulentos de calor latente $(\mathrm{Ft})$ y sensible $(\mathrm{Fg})$ del mar a la masa de aire fría ayudan a una profundización hacia el sur de los frentes fríos que, obviamente, llegarán en mayor número a la región occidental cubana, contribuyendo a una importante disminución en la formación de bajas extratropicales (Fig. 5a).

La segunda hipótesis de trabajo, donde los flujos de calor sensible y latente de la masa de agua caliente, muestran el aumento del intercambio con la de aire menos caliente, aportando un mayor flujo de calor hacia el aire (Fig. 5b). Por lo general, esta situación coadyuva a que la masa de aire frío no invada tanto la región occidental cubana, produciendo un choque entre ambas masas, aumentando la génesis de bajas extratropicales y disminuyendo la llegada de frentes. Es habitual que los frentes fríos que se presentan en esta situación, se disipan en su porción sur aumentando también las lluvias que acompañan a los mismos.

La serie discontinua de las anomalías de la TSM en un punto medio del golfo de México en los eventos ENOS 196970; 1972-73; 1976-77; 1982-83 y 1992 (Bottomley et al., 1990), muestra que los períodos fríos del 68 al 70 y del 7273 se presentaron con algunos pulsos de anomalías positivas que no rebasaron el valor 1 de anomalía en el primero de los casos (Fig. 6), mientras que el total de frentes arribados durante las temporadas invernales del 69-70 y del 72-73 fueron de 30 y 26 frentes, respectivamente (Tabla 1). La temporada mayor de arribo de frentes fríos por la región occidental cu- 


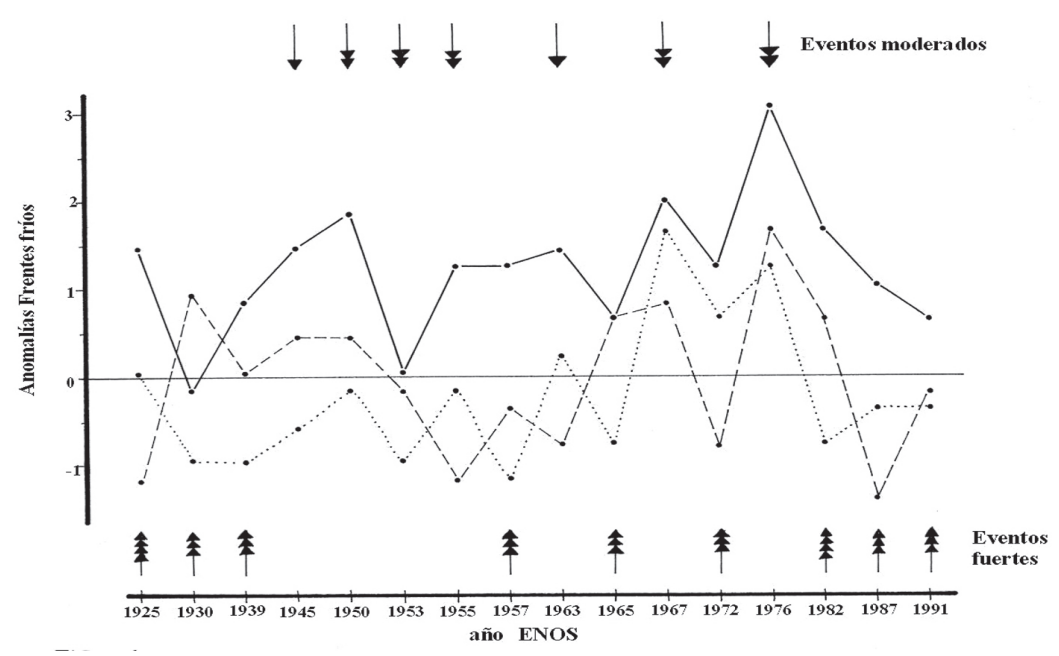

Figura 4. Anomalías de frentes fríos arribados en años alrededor de los eventos ENOS. Las flechas superiores indican los eventos considerados moderados (una o dos flechas) y las flechas inferiores los eventos considerados fuertes (tres o cuatro flechas).

Figure 4. Cold fronts anomalies occured during the ENSO events periods. The upper arrows indicate the events considered as moderate (one or two arrows) and the lower arrows indicate the events considered as strong (three or four arrows).

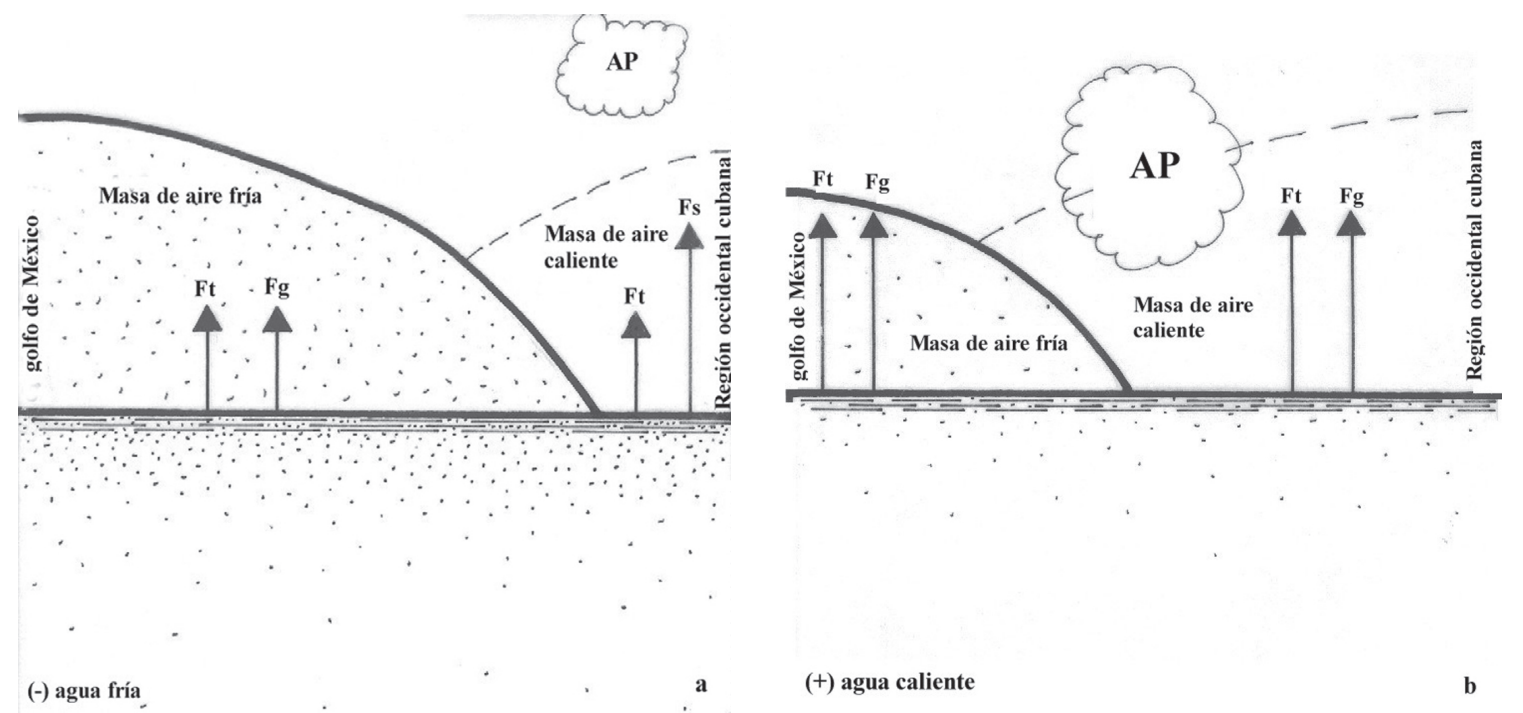

Figura 5. a) Representación esquemática de la hipótesis 1: incremento en las anomalías negativas de la TSM puede provocar poco intercambio de flujos turbulentos en la masa de aire fría, aumentar en el arribo de los frentes a la región occidental, y disminuir sensiblemente la formación de bajas presiones extratropicales en la zona. b) Representación esquemática de la hipótesis 2: incremento en las anomalías positivas de la TSM puede provocar un aumento del intercambio de los flujos turbulentos en la masa de aire fría, coadyuvar a la disminución en el arribo de frentes y aumentar la formación de bajas presiones extratropicales en la zona.

Figure 5. a) Schematic representation of the hypothesis 1: increase of the TSM negative anomalies can cause few exchange of turbulent fluxs in the cold air mass, and increase the arrival fronts to the western region, and diminish the formation of low pressures extratropicales noticeably in the zone. b) Schematic representation of the hypothesis 2: increase of the positive TSM anomalies can cause an increase of the exchange of the turbulent fluxs in the cold air mass, can cooperate to the decrease of flux arrivals and to increase the formation of low pressures extratropicales in the zone. 


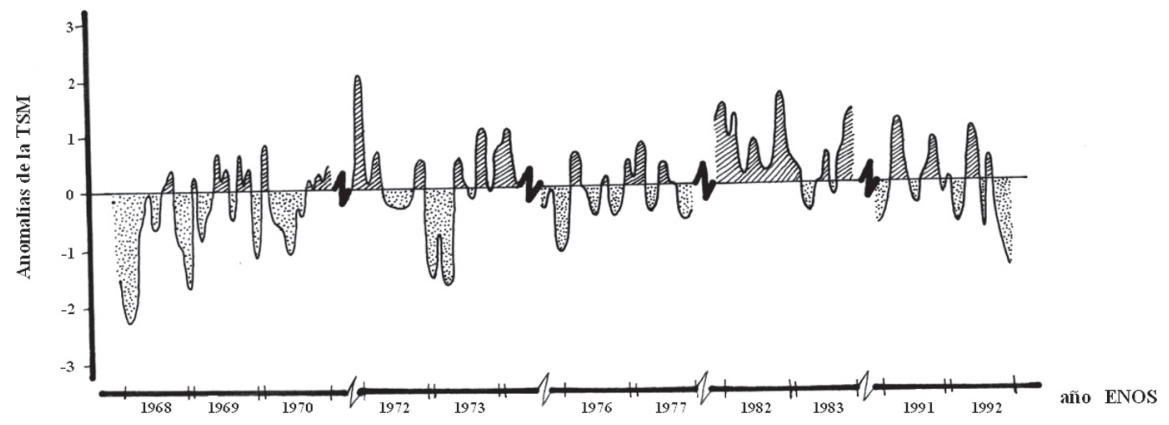

Figura 6. Serie de las anomalías de la TSM en $\operatorname{los} 25^{\circ} \mathrm{N}$ y $88^{\circ} \mathrm{W}$ en el golfo de México, según Bottomley et al. (1990) y Oceanography Summary (1991-92).

Figure 6. Series of the TSM anomalies at $25^{\circ} \mathrm{N} 88^{\circ} \mathrm{W}$ in the Gulf of Mexico, according to Bottomley et al. (1990) and Oceanography Summary (1991-92).

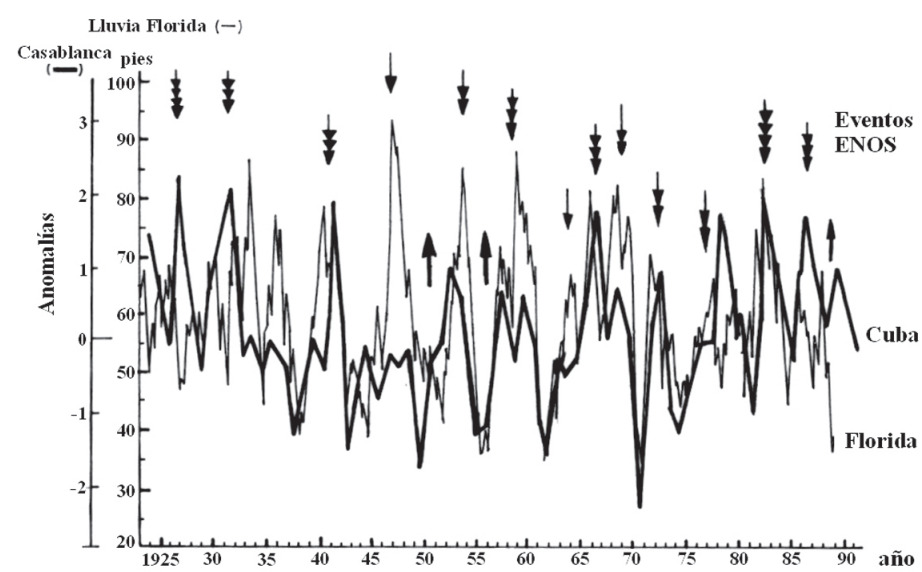

Figura 7. Series cronológicas de lluvia en la región suroriental de la Florida (lower east coast) según Hanson y Maul (1991) y serie de lluvia registrada en la Estación Meteorológica de Casablanca, La Habana, en su promedio de octubre-marzo.

Figure 7. Rain chronological series in the southeastern region of Florida (lower east coast) according to Hanson and Maul (1991) and rain series reported by Meteorological Station of Casablanca, The Havana, average of October-March.
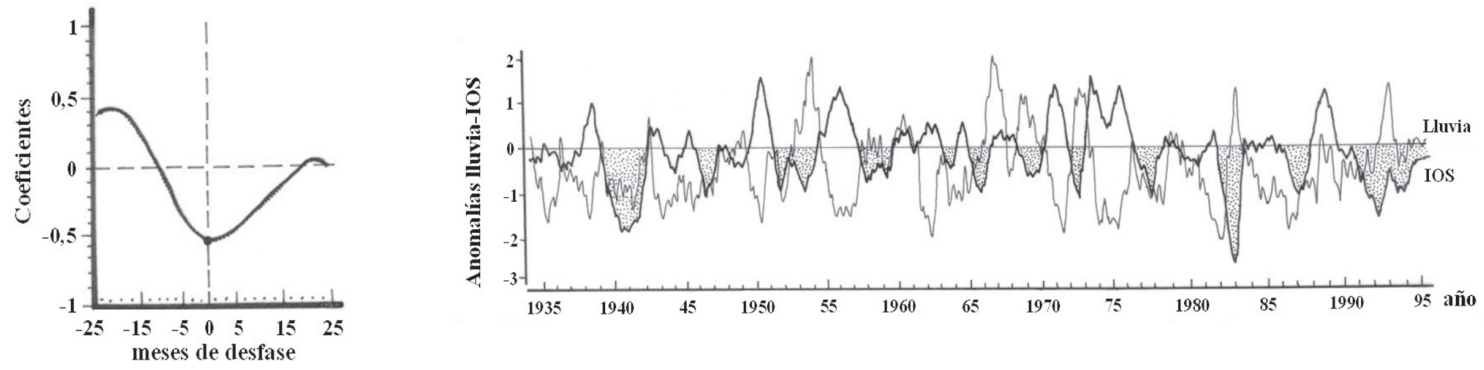

Figura 8. a) Coeficiente de correlación cruzada entre el Índice de Oscilación del Sur y la lluvia. b) Serie cronológica del Índice de Oscilación del Sur y de las anomalías de la lluvia registradas en la Estación Meteorológica de Casablanca, desde 1934 hasta marzo de 1996.

Figure 8. a) Cross correlation coefficient between the South Oscillation Index and rain. b) South Oscillation Index chronological series and rain anomalies reported by Meteorological Station of Casablanca, from 1934 to March of 1996. 
bana ocurrió durante los meses invernales de 197677 (Tabla 1) con 35 frentes, mientras que las anomalías de la TSM según Bottomley et al. (1990) en los años 1976-77 se mantuvo oscilando con anomalías negativas y positivas cercanas a 0 . Por otro lado, las anomalías de la TSM en el evento ENOS 198283 tuvieron un sostenido comportamiento positivo cercano al valor 2 de anomalía. Sin embargo, la temporada invernal se comportó activa con 28 frentes siendo la tercera mayor entrada de frentes en serie que se analiza.

La lluvia acaecida en el suroeste de la península de Florida, ofrecida por Hanson y Maul (1991) y las anomalías de la lluvia en Casablanca muestra una tendencia parecida en ambas series, las cuales son mayores aún en los períodos de poca lluvia o sequías (Fig. 7). Nótese la importante disminución de lluvias durante los años 1949-50, 1962-63 y 196970 , mientras que por lo general, las máximas precipitaciones ocurrieron en eventos ENOS catalogados de fuerte.

El coeficiente de correlación cruzada entre el IOS y las precipitaciones mensuales de Casablanca mostró una mayor correlación sin desfase mensual entre ambas variables, indicando un incremento de lluvias en la región occidental cubana cuando la fase del IOS es negativa (Fig. 8a). El análisis de las series del IOS y la lluvia en la EM de Casablanca muestra períodos lluviosos en la fase negativa del IOS y de sequía en la fase positiva (Fig. 8b). En algunos años, como en la década de los sesenta donde el IOS no marca diferencias extremas, las oscilaciones de la lluvia se comportan siguiendo el patrón de este índice. Por lo general, cuando el IOS es negativo se presentan elevadas temperaturas del mar en la fase Niño 3, antesala de un evento ENOS. El incremento de lluvias en Casablanca obedece al traslado casi simultáneo por la corriente en chorro a 12 $\mathrm{km}$ de altura, de altos cúmulos que se originan en la fase Niño 3 hacia la cuenca del Caribe (Rogers, 1988; Gray y Sheaffer, 1991) y al estado de disipación e inestabilidad que presentan algunos frentes.

Las anomalías promedio de la temperatura del aire para octubre-marzo en la EM de Casablanca desde 1923 hasta 1995-96 según Abel Centella (INSMET, com. pers.), indican que los períodos fríos más importantes se registraron en las temporadas 1929-30, 1930-31 (la más baja reportada), 1939-40; 1940-41; 1950-51, en las temporadas entre 196566 hasta $1969-70$ y el período comprendido entre 1982-83 y 1987-88 (Fig. 9). Los períodos calientes en la región occidental se registraron en las temporadas de $1946-47$; 1947-48; 1958-59; 1971-72 hasta $1974-75$; $1988-89$ hasta $1990-91$ y desde $1993-$ 94 hasta 1994-95. En general, la tendencia de la temperatura del aire ante los eventos ENOS es a disminuir y sólo en los últimos seis eventos reportados en este trabajo, la temperatura mostró una tendencia al incremento en su anomalía.

La Figura 10 muestra el análisis de la temperatura del aire (promedio 1953-1983), con referencia a la ocurrencia de ocho eventos ENOS en Estados Unidos (Namias y Cayan, 1984). Las anomalías de la temperatura promedio se indicaron en tres clases de ocurrencias climatológicas iguales: por encima de lo normal, normal y por debajo de lo normal. En ella se observaron los años 1957-58, 1963-64, 196970 y 1976-77 como inviernos que intensificaron las bajas temperaturas al sureste de Estados Unidos, mientras que en la EM de Casablanca se registraron igualmente temperaturas bajas y máximos de arribo de frentes en esos años. No sucedió así con los eventos ENOS de 1953-54; 1965-66; 1972-73 y 198283 que se reportaron como normales o por encima de lo normal, en el sureste del continente americano y península de Florida. Por su parte, las anomalías de temperatura del aire en la EM de Casablanca para esos años fueron positivas, lo cual indica la similitud existente entre ambas regiones.

La Figura 11 muestra el comportamiento de las anomalías de presión atmosférica promedio octubre-marzo desde 1923-24 hasta 1995-96 en la EM de Casablanca. En ella se señalan oscilaciones anómalas registradas alrededor de 1930 y hasta 1950, además de un período de comportamiento negativo desde aproximadamente 1955 hasta 1973 y desde el 1976 hasta el 1983. El fenómeno ENOS de 1982-83 afectó la presión atmosférica con registros negativos importantes en todas las estaciones meteorológicas del occidente de Cuba (Sosa y Rodríguez, 1992), especialmente en la EM de Casablanca. El registro más bajo ocurrido en 1993-94 se debió principalmente a sistemas de bajas presiones en el golfo de México (Fig. 11).

El coeficiente de correlación cruzada entre el IOS y la presión en Casablanca, señala la existencia de un desfase de 6-7 meses entre ambas variables con un coeficiente de correlación significativamente alto (Fig. 12a). Según este resultado, el IOS podría utilizarse como otro predictor del campo bárico y prever con antelación el estado del tiempo en la región occidental de Cuba. No obstante, los resultados ob- 


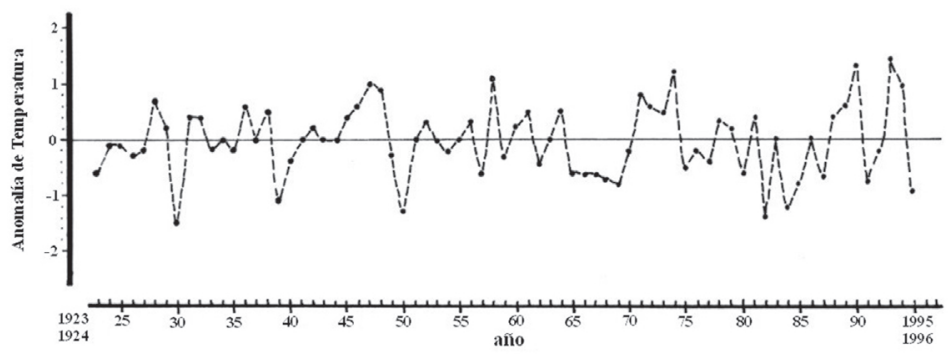

Figura 9. Serie de tiempo de las anomalías de la temperatura del aire media mensual (promedio octubre-marzo) en la Estación Meteorológica de Casablanca desde 1923-24 hasta 1995-96.

Figure 9. Time series of the monthly mean temperature air anomalies (average October-March) by Meteorological Station of Casablanca from 1923-24 to 1995-96.

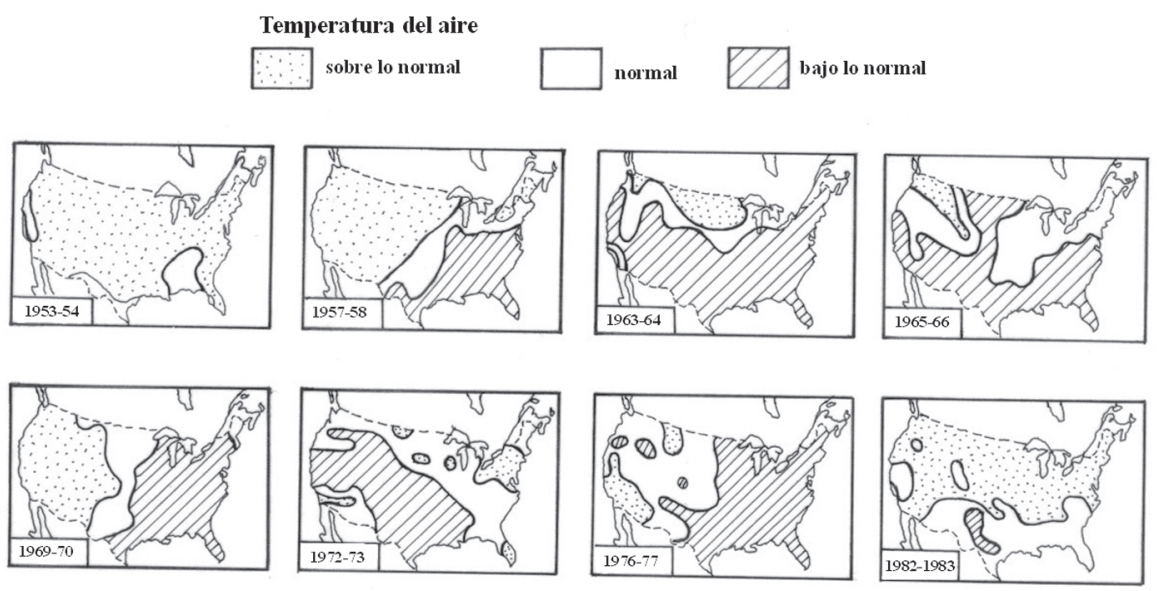

Figura 10. Anomalías de la temperatura superficial del aire en invierno durante 8 eventos ENOS consecutivos en los Estados Unidos, según Namias y Cayan (1984).

Figure 10. Air surface temperature anomalies in winter during 8 consecutive ENSO events in the United States, according to Namias and Cayan (1984).

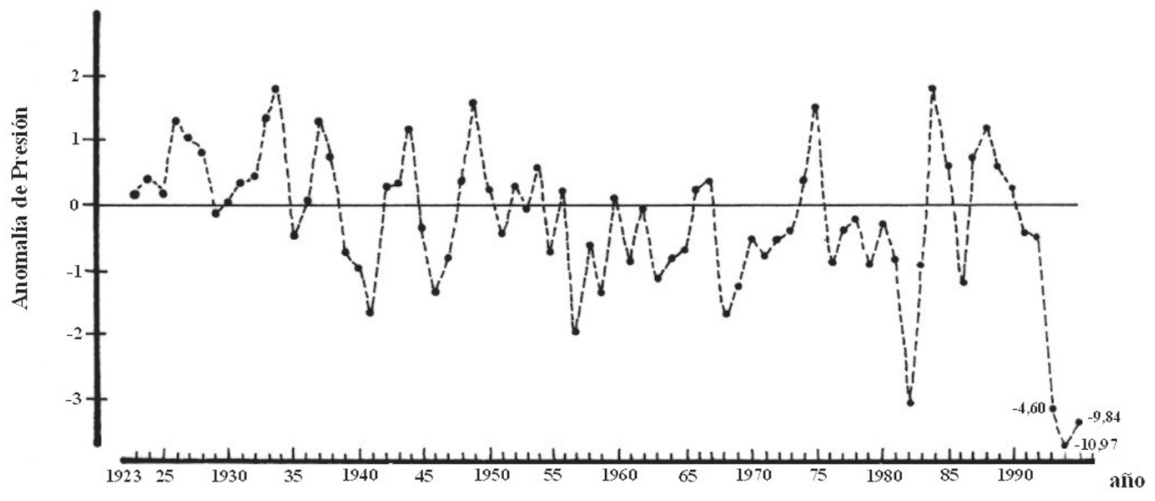

Figura 11. Serie de las anomalías de la presión media mensual en la Estación Meteorológica de Casablanca (promedio octubre-marzo) desde 1923-24 hasta 1995-96.

Figure 11. Monthly mean pressure anomalies series by Meteorological Station of Casablanca (average OctoberMarch) from 1923-24 up to 1995-96. 
tenidos deben tomarse con cautela y se debe establecer un monitoreo constante sobre el IOS, aunque no cabe duda que este aspecto debe estudiarse con mayor profundidad. La relación entre la serie cronológica del IOS y las anomalías de presión atmosférica de 1934 a 1992 (promedios móviles 12 meses), indica que ante la presencia de eventos ENOS en el Pacífico ecuatorial, el comportamiento de la presión atmosférica es a descender, siendo el IOS quien tiende a hacerlo primero con el desfase señalado (Fig. 12b).

La Tabla 2 muestra la serie total de frentes arribados a la región occidental, desde la temporada 192324 hasta 1995-96, el IOS como indicativo de los años ENOS desde 1933-34, la temperatura del aire, la presión atmosférica y las precipitaciones en la EM de Casablanca, todas en su promedio de octubre-marzo.

La Figura 13 muestra el promedio de las anomalías para los frentes fríos, temperatura del aire, presión atmosférica y lluvia en los años alrededor de los eventos ENOS. En ésta, que tiene como objetivo resumir las Épocas Superpuestas, se observa como el promedio en el año de afectación del ENOS (0) es mayor y/o menor según la variable, que en el año previo ó posterior $(-1,+1)$.

Para la aplicación del análisis de Épocas Superpuestas se asumen las hipótesis nulas de las anomalías
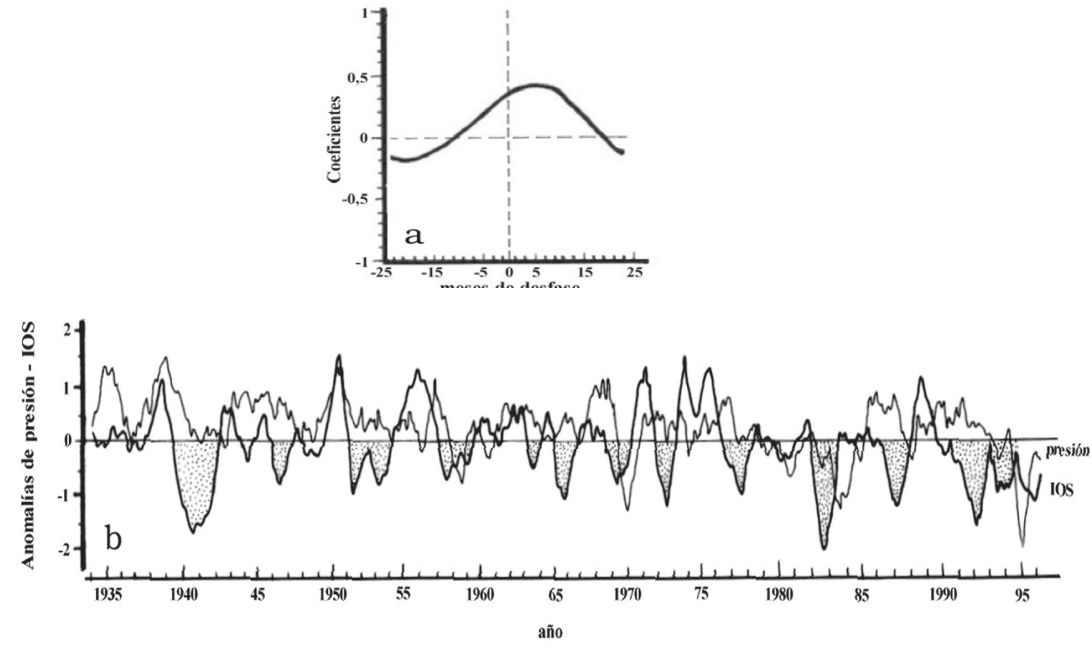

Figura 12. a) Coeficiente de correlación cruzada entre el Índice de Oscilación Sur y la presión atmosférica a nivel del mar. b) Serie cronológica del Índice de Oscilación Sur y de las anomalías de la presión atmosférica registradas en la Estación Meteorológica de Casablanca desde 1934 hasta marzo de 1996.

Figure 12.a) Crossed correlation coefficient between the South Oscillation Index and atmospherical pressure at sea level. b) Chronological series of the South Oscillation Index and atmospherical pressure anomalies reported by Meteorological Station of Casablanca from 1934 to March 1996.
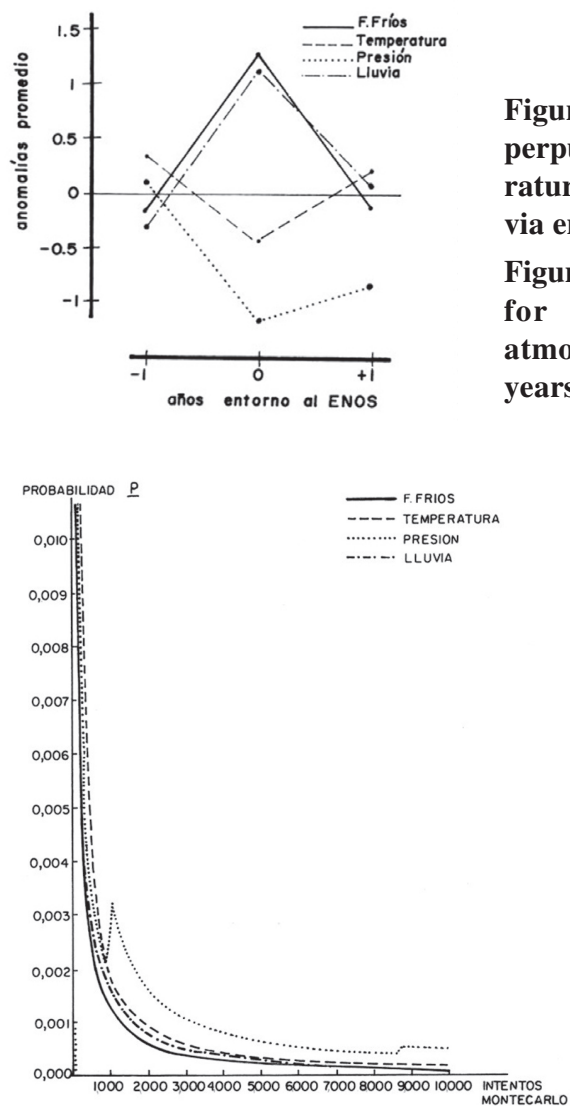

Figura 13. Confección de las épocas superpuestas para los frentes fríos, temperatura del aire, presión atmosférica y lluvia en años entorno a los eventos ENOS.

Figure 13. Superimposition of the periods for cold fronts, air temperature, atmospheric pressure and rain during years around ENSO events.
Figura 14. Nivel de significación estimada $P$ del valor observado $\mathrm{W}$ como una función del número de intentos por simulación aleatoria o método de Monte Carlo.

Figure 14. Significance level estimation $(P)$ of the observed value $(W)$ as a function of the test number for aleatory simulation or Monte Carlo method. 


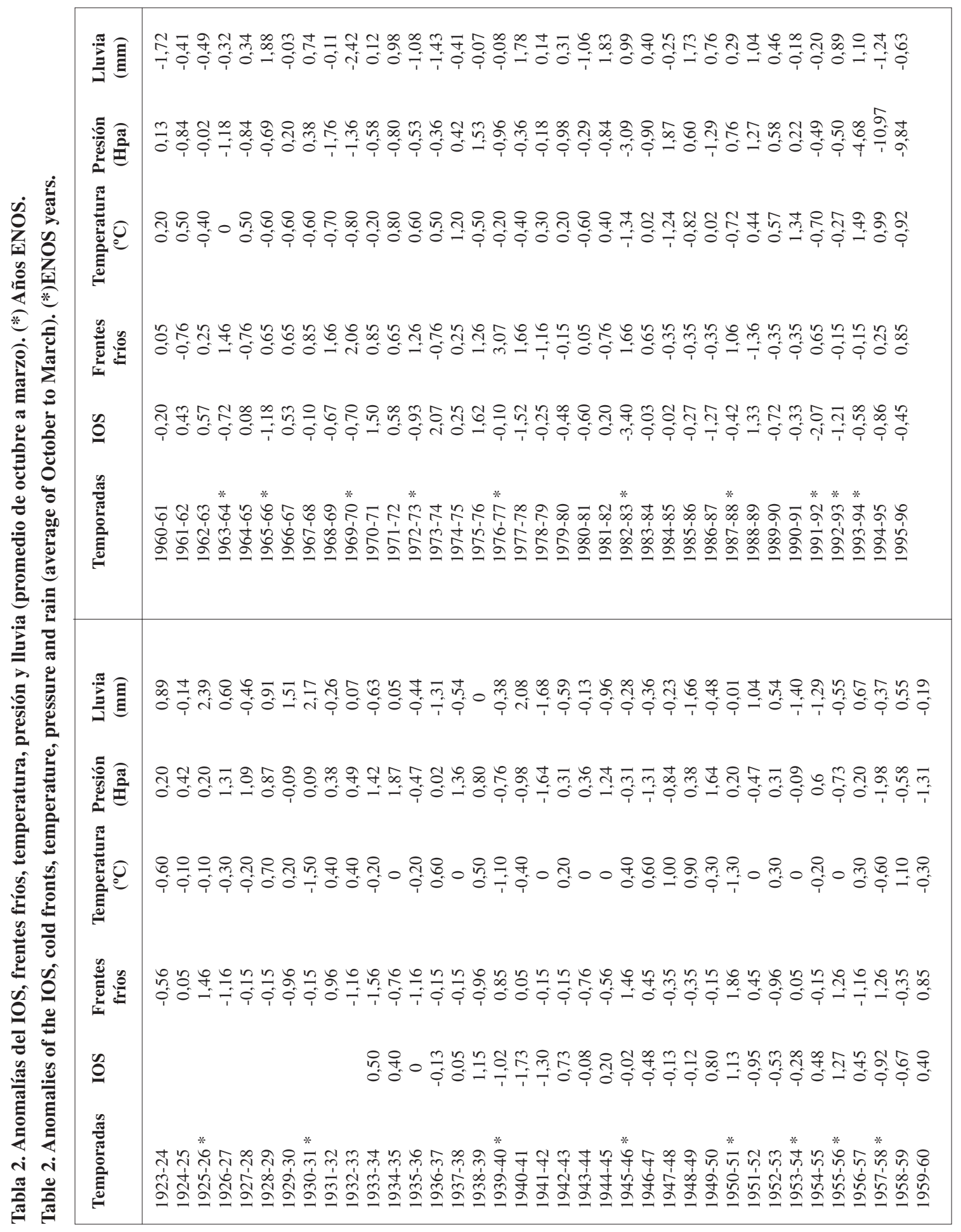


positivas y negativas para cada variable como:

$\mathrm{H}_{0}$ : No hay asociación entre las anomalías positivas de los frentes fríos y de la lluvia con los eventos ENOS.

$\mathrm{H}_{1}$ : No hay asociación entre las anomalías negativas de la temperatura y de la presión atmosférica con los eventos ENOS.

Utilizando el programa modificado del original SEA de Alfonso (1992), entre los máximos de frentes y lluvia y los mínimos de temperatura del aire y presión atmosférica, se encontró que en 10,000 intentos de simulación aleatoria las asociaciones en años donde ocurren los eventos ENOS fueron significativas para una $\mu=0,05(\rho=0,0001 ; 0,0002$; 0,0003 y 0,0001 respectivamente), indicando el establecimiento de una relación muy fuerte de los máximos de entrada de frentes y las variables meteorológicas que le acompañan partir de la ocurrencia de eventos ENOS. A partir de este resultado se rechazan las hipótesis nulas anteriormente planteadas (Tabla 3). La Figura 14 resume el nivel de significación estimada $(\rho)$ del valor observado $(W)$ como una función del número de intentos por simulación aleatoria o Monte Carlo para las anomalías de los frentes, precipitación, temperatura del aire y presión atmosférica en la EM de Casablanca.

\section{DISCUSIÓN}

La manifestación del ENOS en la región occidental cubana contribuye en general a que durante el invierno aparezcan con mayor frecuencia sistemas de bajas presiones en esta área. Como se sabe, la magnitud de los flujos turbulentos la determina el viento, siendo positivo el sentido del flujo si la temperatura del agua es mayor que la del aire. Si se tiene en cuenta que los flujos de calor sensible y latente influyen en las bandas frontales y en su región poste- rior constituyen aportes del océano a las celdas que componen la circulación planetaria (Berlage, 1957), entonces se puede pensar que el aumento en las anomalías positivas TSM en el golfo de México en los ENOS fuertes de 1972-73; 1982-83 y 1991-93 pudieron contribuir a que la energía océano-atmósfera originada al paso de un frente frío presentara valores altos, como consecuencia de la alta acumulación calorífica de las aguas del golfo de México durante esos años. Ello pudo traer como consecuencia, la disipación en la parte sur de algunos frentes que no arribaron a las costas cubanas, que provocó un aumento de la cobertura nubosa con lluvias e inestabilidad de frentes con reiteradas oscilaciones en la región occidental, lo cual causó que el total de frentes arribado no haya sido tan elevado como en realidad debió ocurrir. Es válido destacar que las anomalías de la TSM del golfo de México presentadas en la (Fig. 6) es solo un punto, pero no por ello nos deja de brindar un panorama de lo acontecido.

Lewis y Hsu (1992), demostraron que en eventos ENOS la corriente de lazo ó "Loop Current" constituye una gran fuente consolidada de calor, de la cual un disturbio atmosférico cercano de movimiento lento puede extraer energía y establecerse una fuerte convección en las capas bajas de la atmósfera. Por lo tanto, si el origen del disturbio es una baja extratropical este tiende a fortalecerse, si por el contrario su origen es un sistema frontal, comenzará inobjetablemente su disipación.

Desde el punto de vista oceánico, se manifiesta con una elevación de las anomalías de temperatura superficial y del nivel del mar (Hernández y Puga, 1995), principalmente en la costa norte -que en repetidas ocasiones en combinación con la entrada de frentes fríos fuertes y la fuerza del viento-, han ocasionado funestas penetraciones marinas en el litoral habanero y ocasionado pérdidas en varios sectores de la economía, tales como la cosecha del tabaco,

Tabla 3. Resumen de los parámetros calculados en el Análisis de Épocas Superpuestas (SEA).

Table 3. Summary of the parameters calculated in the Superposed Epoch Analysis (SEA).

\begin{tabular}{|llccccc|}
\hline $\begin{array}{c}\text { Tipo de } \\
\text { análisis }\end{array}$ & Criterio & $\begin{array}{c}\text { Número de } \\
\text { eventos } \\
\text { El Niño }\end{array}$ & $\begin{array}{c}\text { Test } \\
\text { calculado }\end{array}$ & $\begin{array}{c}\text { Número } \\
\text { intentos } \\
\text { Monte Carlo }\end{array}$ & $\begin{array}{c}\text { Ancho } \\
\text { época }\end{array}$ & $\begin{array}{c}\text { Probabilidad } \\
\text { (P) }\end{array}$ \\
\hline W & Frentes & 16 & 11,5 & 10.000 & $-1,0,+1$ & 0,0001 \\
W & Lluvias & 16 & 8,1 & 10.000 & $-1,0,+1$ & 0,0003 \\
W & Temperatura & 16 & 5,7 & 10.000 & $-1,0,+1$ & 0,0002 \\
W & Presión & 16 & 5,6 & 10.000 & $-1,0,+1$ & 0,0001 \\
\hline
\end{tabular}


tomate y caña de azúcar, el turismo y recreación que mayormente se concentra en la región occidental cubana. El saldo final es negativo, principalmente en la salud humana ya que se incrementan las enfermedades catarrales y bronco-aspiratorias (Lecha, INSMET, com. pers.).

Claro está, que la "mecánica" no siempre está acompañada de los mismos efectos, ni de las mismas repercusiones en los eventos y variables meteorológicas y oceánicas que, concatenadamente, dictan las temporadas invernales en Cuba. Más bien se comportan de forma anómala dentro del intervalo de años donde incide el fenómeno ENOS, ya que ningún evento ha sido igual al otro. Estas anomalías por tanto, tendrán una conducta semejante entre los territorios mexicanos y norteamericanos cercanos al golfo, entre ellos la península de Yucatán, Florida y occidente de Cuba.

\section{CONCLUSIONES}

En el período comprendido entre 1923-24 y 199596 existe una asociación directa entre los incrementos del arribo de frentes fríos por la región occidental de Cuba y los años de ocurrencia de eventos ENOS. Las variables meteorológicas más importantes que le acompañan, como temperatura del aire, presión atmosférica y lluvia experimentan variaciones con respecto a su media histórica. El Análisis de Épocas Superpuestas brindó un grado de asociación significativo entre el evento ENOS, el aumento en el arribo de frentes fríos a la región occidental de Cuba y algunos cambios en variables meteorológicas, por lo que se considera una herramienta estadística adecuada y útil.

Los frentes que llegan a la región occidental de Cuba tienden al aumento en años con eventos ENOS moderados (-) y (+) mientras que en años con ENOS fuertes o muy fuertes, también ocurren máximos de entradas de frentes, aunque no tan elevado como en los moderados.

En años ENOS la temperatura superficial del aire promedio octubre-marzo en la EM de Casablanca tiene un comportamiento interanual negativo, con excepción de los períodos calientes de 1945-46, 1972-73, 1982-83 y 1987-88, donde las anomalías fueron positivas.

Se comprobó que la presión atmosférica a nivel del mar en la EM de Casablanca tiene un desfase de aproximadamente 6-7 meses, pudiendo ser ésta una posibilidad de utilizarla como predictora del estado del tiempo en la región occidental cubana.

La lluvia registrada en la EM de Casablanca manifiesta un notable ascenso en la fase negativa del IOS en el período menos lluvioso (o seco), a causa del intenso proceso convectivo que se origina a partir de la irrupción de masas de aire frío, provenientes del continente americano y un sobrecalentamiento superficial del mar en el golfo de México.

\section{AGRADECIMIENTOS}

El autor quiere dejar constancia de su agradecimiento al Dr. George A. Maul, Profesor y Director de la División Marina de la Escuela de Sistemas Ambientales del Instituto Tecnológico de la Florida por su crítica revisión; a los Drs. Eleuterio Yánez y Sergio Palma, y al M.Sc. Patricio Arana de la Universidad Católica de Valparaíso, por su gentileza y entusiasmo por divulgar los resultados de este trabajo. A los Drs. David B. Enfield y Ken Drinkwater (AOML y BIO, respectivamente) por sus valiosos y esclarecedores comentarios en la revisión de este trabajo. Al Dr. Michael H. Glantz, del Grupo de Impacto Social y Ambiental de NCAR, Colorado, por su apreciable gesto en el envío de sus publicaciones y libros. Al Dr. Henry Díaz de la Universidad de Colorado por sus valiosas contribuciones. Igualmente deseo dejar constancia de mi agradecimiento a Dr. W. Quinn (descanse en paz); Dr. V. Neal; Dr. S.G.H. Philander, Dr. V. E. Kousky, Dr. J. Rutllant, Dr. M. Prager; los cubanos Lic. A. Rodríguez del Rey, M.Sc. E. Rodríguez G., A. Betanzos, R. Puga, R. Piñeiro y C. García del Centro de Investigaciones Pesqueras y los Drs. I. Mitrani, M. Sosa, A. Meulenert, Lic. A. Centella, J. P. Osorio del Instituto de Meteorología de Cuba. A todos, gracias.

\section{REFERENCIAS}

Alfonso, A. 1992. SEA: Sistema de análisis de épocas superpuestas. Centro de Investigaciones Pesqueras, La Habana, Manual del usuario, 9 pp.

Berlage, H.P. 1957. Fluctuations of the general atmospheric circulation of more than one year their nature and prognostic value. K. Ned. Meteorol. Inst., Meded. Verh., 69: 1-152. 
Blázquez, L. 1989. Anomalías del nivel del mar en La Habana, Cuba durante el evento "Oscilación del Sur-El Niño" (OSEN), de 1982-83. Instituto de Oceanología. ACC. La Habana, Contrib., 6: 1-12.

Bottomley, M., C.K. Folland, J. Hsiung, R.E. Newell y D.E. Parker. 1990. Global Ocean Surface Temperature Atlas (GOSTA). The Metereological Office-Massachusetts Institute of Technology, 20 pp., 313 plates.

Bradley, R.S., H.F. Díaz, G.N. Kiladis y J.K. Eischeid. 1987. ENSO, signal in continental temperature and precipitation records. Nature, 327 : 497-501.

Dickson, R.R. y J. Namías. 1976. North American influences on the circulation and climate at the North Atlantic sector. Mon. Wea. Rev., 104: 12561265.

Edgington, E.S. 1987. Randomization tests. En: S. Kotz y N. Johnson (eds.). Encyclopedia of statistical sciences. Wiley \& Sons, New York, 7: 530-538.

Enfield, D.B. 1987. Progress in understanding "El Niño”. Endeavour, New Ser., 11(4): 197-204.

Enfield, D.B. 1989. El Niño, past and present. Rev. Geophys., 27(1): 159-187.

Galindo, I y G. Zenteno. 1989. Peculiaridades del fenómeno "El Niño" Oscilación Suriana en la porción noroeste tropical del Océano Pacífico. Com. Tec. Ser. Inv., UNAM, México, 100: 1-4.

Glantz, M.H., R.W. Katz y N. Nichols. 1991. Teleconnections linking worldwide climatic anomalies. Cambridge University Press, New York, pp. 1-12.

Gray, W.M. 1992. Update forecast of Atlantic Seasonal Hurricane Activity for 1992. Clim. Diagn. Bull., 92(7): 44-45.

Gray, W.M. y J.D. Sheaffer. 1991. El Niño and QBO influences on tropical cyclone activity. En: M. Glantz, R. Katz and N. Nicholls (eds.). Teleconnection linking worldwide climate anomalies. Cambridge University Press, New York, pp. 257-284.

Hanson, K. y G.A. Maul. 1991. Florida precipitation and the Pacific "El Niño" 1895-1989. Fla. Sci., 34: 160-168.
Hanson, K., G.W. Brier y G.A. Maul. 1989. Evidence of significant nonrandom behavior in the recurrence of strong El Niño between 1525 and 1988. Geophys. Res. Lett., 16(10): 1181-1184.

Haurtwizt, M. y G.W. Brier. 1981. A critique of the superposed epoch analysis method: its application to solar-weather relations. Mon. Wea. Rev., 109: 2074-2079.

Hernández, B. y R. Puga. 1995. La influencia del fenómeno "El Niño" en la región occidental de Cuba y su influencia en las pesquerías de langosta $(P$. argus) en el golfo de Batabanó. Invest. Mar., Valparaíso, 23: 3-24.

Hoenig, J.M., M.H. Prager y N. Payton. 1989. Computer programs useful for testing hypotheses about recruitment time series. Can. Tech. Rep. Fish. Aqua. Sci., 1713: 1-43.

Horel, J. y M. Wallace. 1981. Planetary scale atmospheric phenomena associated with the Southern Oscillation. Mon. Wea. Rev., 109: 813829.

Law, A.M. y W.D. Kelton. 1982. Simulation modeling and analysis. McGraw-Hill, New York, 784 pp.

Lerch, G. 1984. Diagramas Climáticos de Cuba. ACC, La Habana, 42 pp.

Lewis, J.K. y S.A. Hsu. 1992. Mesoescale air-sea interactions related to tropical and extratropical storms in the Gulf of Mexico. J. Geophy. Res., 97: 2215-2228.

Meehl, G.A. 1978. Tropical teleconections to the seesaw in winter temperatures between Greenland and Northern Europe. NCAR Cooperative Thesis 51. Boulder, CO: National Center for Atmospheric Research, 99 pp.

Namias, J. y D.R. Cayan. 1984. El Niño: Implications for forecasting. Oceanus, 27(2): 41-47.

Oceanographic Monthly Summary. 1991-94. U.S. Dep. of Comm. NOAA, NOS, NWS, NESDIS. Vol. 11-14, Jan.1991 to Dec.1994.

Panofski, W. y G.W. Brier. 1965. Some applications of statistic to meteorology. College of Mineral Industries. The Pennsylvania State University. University Park P.A., 159 pp.

Philander, S.G.H. 1983. El Niño/Southern Oscillation Phenomena. Nature, 302: 295-301. 
Philander, S.G.H. 1985. El Niño and La Niña. J. Atm. Sci., 42 (23): 2652-2662.

Philander, S.G.H. y E.M. Rasmusson. 1985. The Southern Oscillation and El Niño. Adv. Geophys., 28A: 197-215.

Prager, M.H. y J.M. Hoenig. 1989. Superposed Epoch Analysis: A randomization test of environmental effects on recruitment with application to chub mackerel. Trans. Amer. Fish. Soc., 118(6): 608-618.

Prager, M.H. y J.M. Hoenig. 1992. Can we determine the significance of the Key-Event effects on a recruitment time series? A power study Superposed Epoch Analysis. Trans. Amer. Fish. Soc., 121(1): 123-131.

Quinn, W. 1993. The large-scale ENSO event, The "El Niño" and the other important regional features. Bull. Inst. Fr. Étud. Andines, 22(1): 13-34.

Quinn, W. y V.T. Neal. 1989. The historical record of El Niño Events. En: Climate Since A.D.1500. Bradley \& Jones, London, pp. 1-54.

Rasmusson, E.M. 1984. El Niño: The ocean/ atmosphere connection. Oceanus, 27(2): 5-12.

Rasmusson, E.M. y J.M. Wallace. 1983. Meteorological aspects of El Niño/Southern Oscillation. Science, 222: 1195-1202.

Rasmusson, E.M., X. Wang y Ch.F. Ropelewski. 1990. The biennial component of ENSO variability. J. Mar. Syst., 1: 71-96.

Rodríguez, M., C. González y J. Valdés. 1984. Cronología de los frentes fríos que han afectado a Cuba desde la temporada de 1916-1917 hasta la temporada de 1982-1983 (67 temporadas). Reporte de
Investigación No. 9 INSMET. Academia de Ciencias de Cuba, 15 pp.

Rogers, J.C. 1988. Precipitation variability in the Caribbean and Tropical Americas associated with the Southern Oscillation. J. Climate, 1: 172-182

Ropelewski, CH.F. y M.S. Halpert. 1987. Global and regional scale precipitation patterns associated with the "El Niño" Southern/Oscillation. Mon. Wea. Rew., 115: 1606-1626.

Rutllant, J.C. 1985. Algunos aspectos de la influencia climática, a nivel mundial y regional, del fenómeno El Niño. Invest. Pesq., Chile, 32: 9-17.

Sosa, M. y A. Rodríguez. 1992. Variaciones temporales de la presión atmosférica en Casablanca. Rev. Cub. Met., 15(1): 29-33.

Troup, A. J. 1965. The "Southern Oscillation". Qtr. J. Roy. Met. Soc., 91: 490-506.

van Loon, H. y J. C. Rogers. 1978. The seesaw in winter temperatures between Greenland and Northern Europe. Part I. General Description. Mont. Wea. Rew., 108(3), 296-310.

Walker, G.T. 1923. World weather. I. Mem. Indian. Met. Dep., 24: 75-131.

White, M.E. y M.W. Downton. 1991. The shrimp fishery in the Gulf of Mexico: relation to climate variability and global atmospheric patterns. En: M. Glantz, R. Katz and N. Nicholls (eds.). Teleconnection linking worldwide climate anomalies. Cambridge University Press, New York, pp. 429-492.

Zebiak, S.E. y M.A. Cane. 1987. A model El NiñoSouthern Oscillation. Mon. Wea. Rev., 115(10): 2262-2278. 


\section{ANEXO 1}

\section{Breve explicación del Análisis de Épocas Superpuestas}

El Análisis de Épocas Superpuestas o Superposed Epoch Analysis (SEA) es una técnica no paramétrica que se puede usar para hacer pruebas de significación estadística entre eventos ambientales discretos y series de tiempo. Como la significación estadística es determinada por pruebas aleatorias, el SEA no cuenta con los requisitos usuales (muestras aleatorias, homogeneidad de varianza, independencia de observaciones, etc.), de las pruebas paramétricas. En estos términos, el método se puede usar cuando el análisis de regresión, la correlación o la prueba-t sean inapropiados.

\section{Desarrollo de la hipótesis nula y la prueba estadística}

El primer paso en el uso del SEA, como en cualquier prueba estadística, es la formulación de la hipótesis nula y la prueba estadística. Para esto se necesita determinar la distribución de la prueba estadística cuando la hipótesis nula es verdadera.

Ho: Existe asociación entre un caso extremo de un evento ambiental y los datos de la serie de tiempo. (Se puede definir una hipótesis alternativa).

\section{La superposición de épocas}

Para construir las épocas superpuestas, se tiene que definir los años de eventos claves, como los años donde el evento ambiental tuvo un comportamiento crítico. Los datos para la serie de tiempo se organiza en orden cronológico. La selección del año del evento clave se realiza sobre la base de un evento cualitativo, por ejemplo el año de ocurrencia del evento del Niño ó el paso de un huracán. Los años alrededor del año del evento clave se llaman años de fondo. A continuación se da un ejemplo de organización de los datos para el SEA.

\begin{tabular}{|c|c|c|c|}
\hline Años & Evento & (1) & Datos (2) \\
\hline 1900 & 0.02 & 0.354 & Años de fondo \\
\hline 1901 & 0.01 & 0.563 & \\
\hline 1902 & $0.40 *$ & 0.341 & Año de evento clave \\
\hline 1903 & 0.23 & 0.189 & \\
\hline 1904 & 0.03 & 0.218 & Años de fondo \\
\hline
\end{tabular}

(1) En este caso, se puede considerar como ejem- plo, que el evento puede ser el nivel del mar y el año del evento clave (*), aquél en el cual el nivel sobrepasó el doble de la media.

(2) Indice de reclutamiento de una especie de peces.

En este caso la hipótesis nula (Ho) sería:

Ho: La supervivencia tiende a ser mayor cuando el nivel del mar es muy alto.

$\mathrm{El}$ ancho de la época, generalmente, se escoge igual a 4 es decir 2 años antes y 2 años después del año del evento clave, para el caso que se consideren las dos caras. De esta misma forma, se puede considerar que la época tiene una sola cara y solo se tomarían en cuenta los efectos en los años de fondo posteriores al evento. La selección de estos valores depende del estudio que se desee realizar.

\section{Prueba estadística}

El estudio para tomar la hipótesis nula se realiza con cualquiera de las pruebas siguientes:

\section{a) Prueba estadística D = E - B}

Donde D es la diferencia entre la media para los años de eventos claves E y B la media en los años de fondo.

\section{b) Prueba estadística $\mathrm{T}=(\mathrm{E}-\mathrm{B}) / \mathrm{S}$}

Donde $\mathrm{S}$ es la desviación estándar. Esta es calculada como una prueba ordinaria del test t-Student.

c) Prueba estadística $\quad \mathrm{W}=\overline{\mathrm{d}}\left(\mathrm{N}_{\mathrm{B}}\right)^{1 / 2} / \mathrm{S}_{\mathrm{w}}$

Donde $\overline{\mathrm{d}}$ es la media de todas las diferencias pares entre el índice para años de eventos claves i y el índice para cada año en su alrededor.

$\left(\mathrm{B}_{\mathrm{ij}} ; \mathrm{E}_{\mathrm{i}}(-2,-1,1,2)\right)$ más precisamente se define $\overline{\mathrm{d}}$ como:

$$
\overline{\mathrm{d}}=\frac{1}{\mathrm{~N}_{\mathrm{B}}} \sum_{\mathrm{i}=1}^{\mathrm{Ni}} \sum_{\mathrm{j}=1}^{\mathrm{n}_{\mathrm{j}}}\left(\mathrm{E}_{\mathrm{i}}-\mathrm{B}_{\mathrm{ij}}\right)
$$

Sw es una medida de dispersión (su definición corta).

Esta prueba es una análoga al test t-Student calculado para pares de datos. Cada año del evento clave es comparado con sus respectivos años de fondo. Aquí se pueden usar los años de anti-eventos claves, para los cuales se introduce una variante del SEA, el análisis de eventos opuestos considerando otra hipótesis, como:

Ho: Los datos de la serie tienden a ser mayores en los años de eventos claves positivos y menores 
en los de eventos claves negativos.

Prager y Hoenig (1992), hicieron un estudio sobre el poder de cada una de estas pruebas estadísticas y llegaron a la conclusión que con el SEA, las pruebas estadísticas más poderosas son la $\mathrm{D}$ y W. En la simulación de un conjunto de datos con tendencia lineal, D fue la más poderosa, excepto cuando tenía un comportamiento extremo o muy fuerte. En el resto de los casos la prueba W demostró ser la prueba estadística más poderosa.

\section{Determinación de la distribución nula de la prueba estadística}

En una prueba de significación, se decide si el valor observado de la prueba estadística es suficientemente extremo como para rechazar la hipótesis nula para el nivel de significancia escogido. Para tomar esta decisión se debe conocer el valor calculado de la prueba estadística. Para una prueba paramétrica, la distribución nula es usualmente obtenida de una tabla, para ese análisis se obtiene de pruebas aleatorias.

La simulación de Monte Carlo es usada para determinar la distribución nula de una prueba estadística para un grado de precisión arbitrario. Repetida y aleatoriamente se seleccionan las posiciones para los años de eventos claves (y sus correspondientes años de fondo), computándose el resultado de la prueba estadística seleccionada para cada conjunto.
El estimado $\mathrm{P}$ de las pruebas realizadas se calcula como $(\mathrm{x}+1) /(\mathrm{v}+1)$ donde $\mathrm{v}$ es el total de intentos de Monte Carlo y x es el total de pruebas para las cuales la prueba estadística resultó mayor que el valor observado para esa prueba.

Es difícil saber a priori cuantas pruebas o ensayo Monte Carlo se requiere efectuar para obtener una precisión adecuada en los resultados. Edgington (1987) ha publicado fórmulas para obtener con un 99\% de confianza el intervalo de la probabilidad a partir de un número dado de ensayos y una $\mathrm{P}$ dada; estas fórmulas pueden proporcionar alguna guía.

Puede obtenerse un estimado de $\mathrm{P}$ después de un número de ensayos dados, mediante el método de medias por lotes (Law y Kelton, 1982). Una tercera técnica que se puede utilizar, es graficar la $\mathrm{P}$ estimada de cada prueba, contra el número de ensayos si los resultados se han estabilizado dentro de los números especificados de ensayos o pruebas.

Finalmente, se debe señalar una importante observación sobre inferencia estadística, incluyendo el SEA, que es que la hipótesis debe ser formulada antes de la inspección de los datos de respuesta. A pesar de esta limitación, el SEA es un método poderoso para obtener niveles de significación en series de tiempo autocorrelacionadas. 